\title{
Laser Assisted Imprint of Silicon Nanostructure with Good Crystal
}

\section{Quality}

\author{
Eih-Zhe Liang, ${ }^{\mathrm{a}}$, Zhao-Ren Huang ${ }^{\mathrm{a}}$, Ching-Fuh Lin ${ }^{\mathrm{a}, \mathrm{b}}$, and Chieh-Hsiung Kuan ${ }^{\mathrm{b}, \mathrm{c}}$ \\ ${ }^{a}$ Graduate Institute of Electro-optical Engineering, ${ }^{b}$ Department of Electrical Engineering and \\ Graduate Institute of Electronics Engineering, ${ }^{c}$ National Taiwan University Center for Information \\ and Electronics Technologies, National Taiwan University, Republic of China
}

\begin{abstract}
Excimer laser assisted imprint technology is implemented to create nanostructure on silicon substrate. Patterns with 25-nm feature sizes are successfully fabricated on quartz mold using electron beam lithography for nano-imprint. Transfer of nano-meter features to to silicon is experimented. Parameters for optimal imprint are investigated. With a peak light intensity of $1 \sim 2 \mathrm{~J} / \mathrm{cm}^{2}$, imprint pressure of $10 \sim 100 \mathrm{~g} / \mathrm{cm}^{2}$, and high vacuum at $10^{-6}$ torr, nano-imprint has very good results. The carrier lifetimes before and after laser-assisted imprint are studied and discovered to vary from 1818 us to 640 us. This small damage of Si substrate is attributed to recrystallization of silicon.
\end{abstract}

Index Terms - Excimer laser, silicon, nanotechnology, charge carrier lifetime.

\section{INTRODUCTION}

Imprint of nanostructure by high energy pulsed laser is becoming an important process in nanotechnology [1]. Its advantages are massive production of nanostructures on large substrate with reusable master molds. This reduces cost and improves productivity compared to repetitive creation of nanopatterns by electron beam lithography.

The imprint technique is separated into two categories. One is the transfer of nanopatterns on the master mold to resist and applying dry etch to further transfer to underlayer or substrate $[1,2]$. The other is to direct transfer nanopattern on mold by melting the underlayer [3]. This greatly reduces process steps and avoids difficult anisotropy control of dry etch.

Application of laser assisted imprint to creation of silicon nanostructure is important in the future to IC foundry. It is precise and simple in this way to make ridge channel in electronic devices like FinFET [4] and to make isolation islands in quantum devices like single electron transistor [5]. Optical components on silicon with nanostructures of photonic bandgap [6] and subwavelength transmission [7] can be also included in this application field.

The other great advantage of laser assisted imprint is reservation of substrate quality by the point of recrystallization from silicon substrate. In all of these subtle electronic and optical devices, quality of silicon nanostructure influences charge carrier mobility and lifetime and has significant impact on their performance. It also avoids the damage caused by dry etch for functional nanostructure for solar cell application.

In this work, we use quartz mold and directly imprint silicon substrate by excimer laser with peak light intensity up to $2 \mathrm{~J} / \mathrm{cm}^{2}$. Patterns with $25-\mathrm{nm}$ feature sizes are successfully fabricated on quartz mold using electron beam lithography. $100 \mathrm{~nm}$ linewidth ridge pattern on quartz mold can be transferred successfully to silicon. Difficulties and solutions of decananometer structure of master quartz mold by e-beam lithography are also addressed. Conditions of imprint of silicon, like imprint pressure, optical intensity and ambient, are characterized. Charge carrier lifetime, an indicator to crystal quality, is found to change merely from 1818 us to 640us, compared to detrimental change to 35 us by dry etch. This is the consequence of recrystallization of silicon without forming defect and prevention from oxygen dissolution in high vacuum.

\section{PRINCIPLE OF LASER ASSISTED IMPRINT}

The principal process of laser-assisted imprint by high energy pulse laser melting is depicted in Fig.1. The first step is to create nano-patterns on the quartz master mold by deep UV or electron beam lithography, and subsequent dry etch of quartz. The patterned mold is attached to silicon surface, and silicon wafer is placed in a chamber for illumination of laser beam, as shown in Fig. 1a).

The degree of contact between patterns on quartz and silicon surface is controlled by applying pressure on the quartz mold. The applied pressure for intimate contact is in the range of $10 \sim 100 \mathrm{~g} / \mathrm{cm}^{2}$. The chamber ambient is pumped to base ambient pressure of $10^{-6}$ torr. This avoids oxidation of silicon during melting and interference of illumination from dust particles. 
The laser source is $\mathrm{KrF} 248 \mathrm{~nm}$ line with $25 \mathrm{~ns}$ duration and its illumination intensity is tunable up to $2 \mathrm{~J} / \mathrm{cm}^{2}$ on samples. Homogeneity of the laser beam is maintained to have variation no larger than $10 \%$ within $4 \times 4 \mathrm{~mm}^{2}$. The intensity threshold of melting silicon is estimated under $1 \mathrm{~J} / \mathrm{cm}^{2}$, melting duration is within $50 \mathrm{~ns}$, and the melting depth is $100 \mathrm{~nm}$ per shot [8]. The imprint depth is estimated to be the same for each shot and the total required depth may be controlled by intensity tuning or multiple shots.

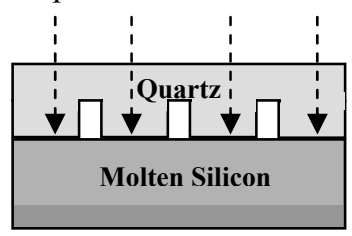

a)

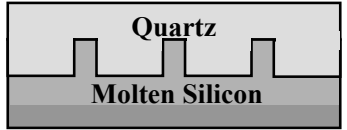

b)

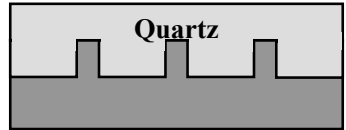

c)

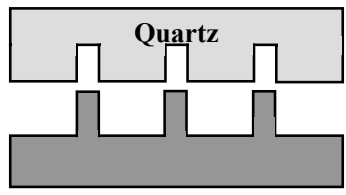

d)
Fig. 1 Principles of laser assisted imprint. a) Quartz mold is attached to silicon surface and Si surface is molten by high pulse energy laser. b) quartz mold is imprinted into molten silicon c) $\mathrm{Si}$ surface recrystallizes, d) quartz mold and silicon are separated.

During melting of silicon, patterns on the quartz mold press into $\mathrm{Si}$ due to mechanical pressure. Silicon then recrystallizes back from crystalline substrate after dispatch of heat, as shown in Fig. 1b). Question may arise if quartz mold is molten and dissolute with silicon. Experiments show that it isolated from melting silicon whether it melts or not by examination of intact patterns on quartz mold after imprint. The quartz mold is then removed from silicon substrate after recrystallization is finished, as shown in Fig. 1c) and Fig. 1d). It is simply separated by release of pre-asserted pressure. Disrupt of either quartz or silicon nanostructure by random force during separation is yet to happen in $100 \mathrm{~nm}$ scale.

\section{CREATION OF QUARTZ DECANANOMETER STRUCTURE}

Details in creation of decananometer structures on quartz are as follows. Electron-beam lithography is commonly used to create nanopatterns on quartz. However, charging problem in projection of electron to resist exists. Two techniques are applied to solve this problem. As shown in Fig. 2a), a conductive polymer is spin-coated onto e-beam resist. This provides grounding of current from projection spots, but large pitch up to $100 \mathrm{~nm}$ is required. This method takes advantage of polymer dissolution in water by modification with hydroxyl group.
Another technique is to deposit conductive silicon or metal before the resist application, as shown in Fig. 2b). After e-beam exposure and development of resist, the pattern is then transferred to conductive under-layers by reactive ion etch. This could result in smaller pitch down to $50 \mathrm{~nm}$. In both approaches, spot size is controlled by exposure beam size, current dose, and development time.

The production of decananometer pore pattern on quartz is shown in Fig. 2c) and Fig. 2d) with respective method. E-beam resist of $100 \mathrm{~nm}$ thickness (Zeon chemicals) is used and whole lithography takes place in ELS-7500EX (Elionix). Conductive polymer is charge dissipating agent of $10 \mathrm{~nm}$ thickness (Showa Denko) and is rinsed away before development. Conductive underlay is silicon of $50 \mathrm{~nm}$ thickness. The minimum spot size achieved is $25 \mathrm{~nm}$ in diameter.

After the resist pattern is created, chromium lift-off may take place to reverse the trench/pore pattern to ridge/pillar pattern. The nano-patterned quartz master mold is etched by $\mathrm{CHF}_{3} / \mathrm{Ar}$ in RIE chamber. Typically $150 \mathrm{~nm}$ to $300 \mathrm{~nm}$ height is generated and the mold is ready for imprint use.
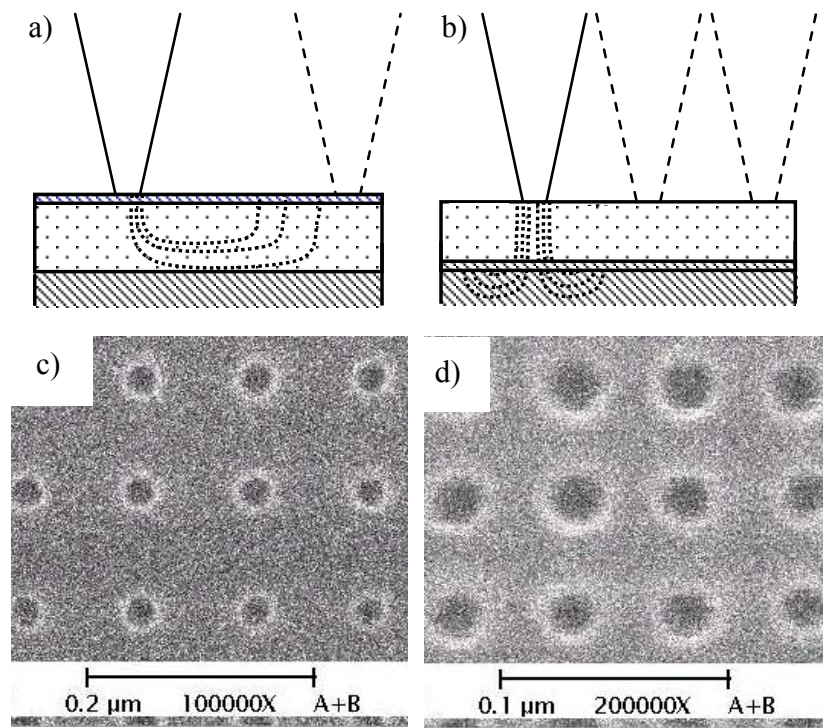

FIG 2. E-beam spot and pitch restriction of a) conductive polymer on resist and b) metal or silicon underlayer beneath resist. c) Attainable 100nm pitch of method a and d) $50 \mathrm{~nm}$ pitch of method b. Both spots are of $25 \mathrm{~nm}$ diameter.

\section{IMPRINT RESULTS}

Although decananometer structure on quartz is readily created, imprint on silicon is restricted to $100 \mathrm{~nm}$ scale in this report and improvement is further investigated. In Fig. 3 a) it is quartz ridge pattern with $100 \mathrm{~nm}$ linewidth and $1 \mu \mathrm{m}$ pitch. The roughness or variation of linewidth is less than $5 \%$ in the observable area of all ridges span as 
long as 600um.

This pattern is imprinted onto silicon by laser-assisted imprint with $2 \mathrm{~J} / \mathrm{cm}^{2}$ light intensity and $70 \mathrm{~g} / \mathrm{cm}^{2}$ pressure. The resulted pattern is $250 \mathrm{~nm}$ linewidth trench on silicon. The broadening of linewidth on silicon comes from trapezoidal shape of ridge on quartz. The imprinted trench is straight over 600um field with no observable distortion. The variation in linewidth is $5 \%$ and proves fidelity in hundred nanometer pattern transfer by imprint.
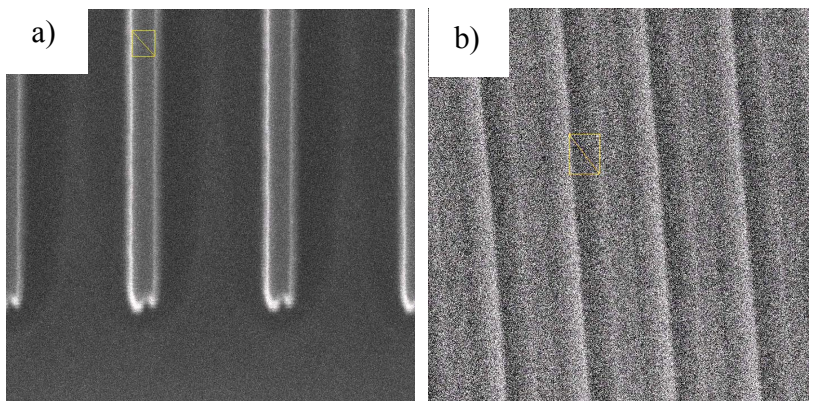

FIG 3. a) Ridge of $100 \mathrm{~nm}$ linewidth and $1 \mu \mathrm{m}$ pitch on quartz mold and b) imprinted silicon with the same pitch.

\section{IMPRINT DAMAGE CHARACTERIZATION}

Characterization of damage from imprinting nanostructures asserts extendibility of laser-assisted imprint to many applications sensitive to charge carrier mobility and lifetime. It is demonstrated by measurement of charge carrier lifetime before and after imprint process. The lifetime is extracted by laser-excited microwavereflection photo-conductive-decay (MWPCD). Surface charge effect on silicon wafer is removed by immersion in iodine/methanol solution. The excitation power density is up to $700 \mathrm{~kW} / \mathrm{cm}^{2}$ using Nd-YAG SHG $532 \mathrm{~nm}$ pulsed laser.

As shown in Fig. 4, the original lifetime of p-type float-zone growth silicon wafer is measured as $1818 \mu \mathrm{s}$. The lifetime drops to $981 \mu \mathrm{s}$ after one-shot illumination of $2 \mathrm{~J} / \mathrm{cm}^{2}$ laser pulse. It drops to $640 \mu \mathrm{s}$ after imprint with quartz mold. This drop may be caused by oxygen absorption during silicon recrystallization. All these figures of lifetime are typical for solar cell application. It is much better than $35 \mu \mathrm{s}$ after $\mathrm{RIE}$ etch with $\mathrm{SF}_{6} / \mathrm{O}_{2}$ species.

This is the first report on small-damage attribute of laser-assisted imprint of nanostructures on silicon substrate. It is intuitive in view point of silicon recrystallization from high quality substrate. It has been reported that silicon after high pulse laser illumination remains single crystalline only if regrowth velocity of liquid-solid interface is between 2 to $10 \mathrm{~cm} / \mathrm{s}$ [9]. This condition can be sustained by maintaining illumination intensity to $1 \sim 2 \mathrm{~J} / \mathrm{cm}^{2}$. We have taken into account another factors like high vacuum ambient in minimization of oxygen dissolution in molten phase of silicon.

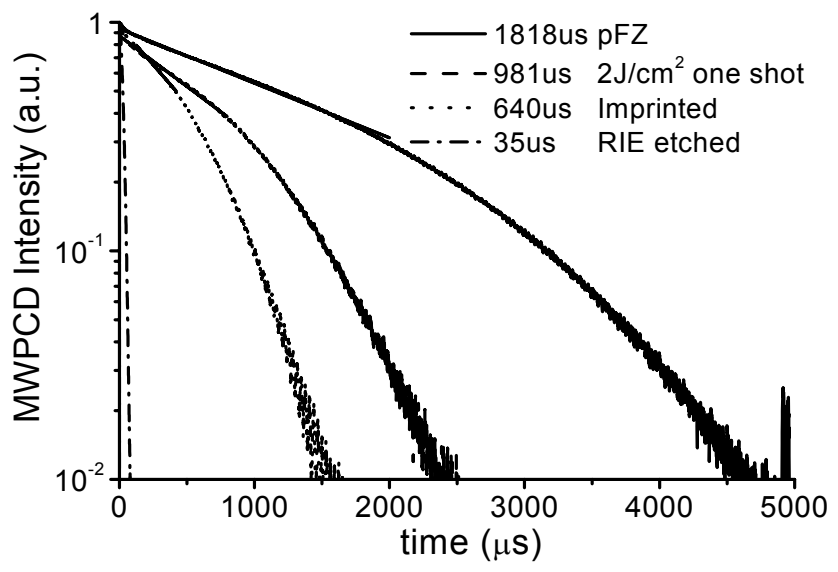

FIG 4. MWPCD curve to obtain charge carrier lifetime before any treatment (solid), after pulse laser illumination (dash), after imprint with nanopatterned quartz mold (dot), and with RIE etch (dash dot)

After assertion of silicon quality by laser melting, the imprinted nanostructure of silicon is proved to be only slightly damaged. Compared with high damage of dry etch, which has at least 20 times more recombination trap generated, the laser-assisted direct imprint technology gives much better crystal quality. MWPCD has sensitive detection of $30 \mathrm{~nm}$ damage layer of silicon surface by dry etch [10], so the $100 \mathrm{~nm}$ scale imprinted structure has no ambiguity in high quality with substrate.

\section{CONCLUSION}

Small damage laser-assisted imprint is successful in creation of $100 \mathrm{~nm}$ scale nanostructure on silicon substrate. Quartz mold is used and advanced electron beam lithography for creation of decananometer structure is implemented. Patterns with 25-nm feature sizes on quartz mold are successfully fabricated using electron beam lithography. Using the excimer laser with peak light intensity up to $2 \mathrm{~J} / \mathrm{cm}^{2}, 100 \mathrm{~nm}$ linewidth ridge pattern on quartz mold can be transferred successfully to silicon. Imprint pressure of $10 \sim 100 \mathrm{~g} / \mathrm{cm}^{2}$, optical intensity within $1 \sim 2 \mathrm{~J} / \mathrm{cm}^{2}$ and vacuum $10^{-6}$ torr are optimized condition for small-damage imprint. Charge carrier lifetime changes merely from 1818 us to 640us, compared to detrimental change to 35 us after dry etch. This is attributed to recrystallization of silicon from substrate upward to surface and prevention from oxygen dissolution in high vacuum. 


\section{ACKNOWLEDGEMENT}

The authors wish to acknowledge support of National Science Council under contract number NSC 93-2120-M-002-011 and NSC 93-2112-M-002-032.

\section{REFERENCES}

[1] S.Y. Chou, P.R. Krauss, and P.J. Renstrom, "Imprint lithography with 25-nanometer resolution," Science 272, 85-87 (1996)

[2] M.D. Austin, H. Ge, W. Wu, M. Li, Z. Yu, D. Wasserman, S.A. Lyon, and S.Y. Chou, "Fabrication of $5 \mathrm{~nm}$ linewidth and $14 \mathrm{~nm}$ pitch features by nanoimprint lithography," Applied Physics Letters 84, 5299-5301 (2004)

[3] S.Y. Chou, C. Keimel, and Jain Gu, "Ultrafast and direct imprint of nanostructures in silicon" Nature 417, 835 (2002)

[4] X. Huang,W.C. Lee, C. Kuo, D. Hisamoto, L. Chang, J. Kedzierski, E. Anderson, H. Takeuchi, Y.-K. Choi, K. Asano, V. Subramanian, T.-J. King, J. Bokor, and C. Hu, "Sub-50 nm P-Channel FinFET," IEEE Trans. Elec. Dev. 48, $880,(2001)$..

[5] K. Zhuang, L. Guo, and S.Y. Chou, "Silicon single-electron quantum-dot transistor switch operating at room temperature," Applied Physics Letters 72, 1205 (1998)

[6] M. Notomi, A. Shinya, E. Kuramochi, I. Yokohama, C. Takahashi, K. Yamada, J.-I. Takahashi, T. Kawashima, and S. Kawakami, "Si-based photonic crystals and photonic-bandgap waveguides," IEICE Transactions on Electronics E85-C, 1025 (2002)

[7] Z. Yu, W. Wu, L. Chen, and S. Y. Chou, "Fabrication of large area $100 \mathrm{~nm}$ pitch grating by spatial frequency doubling and nanoimprint lithography for subwavelength optical applications," Journal of Vacuum Science and Technology B: Microelectronics and Nanometer Structures 19, 2816-2819 (2001)

[8] B.C. Larson, J.Z. Tischler, and D.M. Mills, "Nanosecond resolution time-resolved $\mathrm{x}$-ray study of silicon during pulsed-laser irradiation," Journal of Materials Research 1, 144-154 (1986)

[9] A.G. Cullis, H.C. Webber, and N.G. Chew, "Ultra-fast melting and solidification behavior of amorphous and crystalline silicon," Materials Research Society Symposia Proceedings 23, 105-110 (1984)

[10]E.-Z. Liang, C.J. Huang, and C.F. Lin, "20nm Silicon nanorods fabricated by reactive ion etch," 4th IEEE Conference on Nanotechnology TU-P42, (2004) 